\title{
Feasibility of adjuvant chemotherapy with S-1 consisting of a 4-week administration and a two-week rest period in patients with completely resected non-small cell lung cancer
}

\author{
SHUNSUKE OKUMURA ${ }^{1}$, TAKAAKI SASAKI ${ }^{1}$, KAZUHIRO SATOH $^{1}$, MASAHIRO KITADA ${ }^{1}$, \\ ATSUSHI NAGASE $^{2}$, EIJI YATSUYANAGI ${ }^{3}$ and YOSHINOBU OHSAKI $^{1}$ \\ ${ }^{1}$ Respiratory Center, Asahikawa Medical University, Asahikawa, Hokkaido 0788510; \\ ${ }^{2}$ Department of Respiratory Surgery, National Hospital Organization, Asahikawa Medical Center, \\ Asahikawa, Hokkaido 0708644; ${ }^{3}$ Department of Respiratory Surgery, National Hospital Organization, \\ Obihiro Hospital, Obihiro, Hokkaido 0808518, Japan
}

Received June 6, 2012; Accepted July 30, 2012

DOI: $10.3892 / \mathrm{mco} .2012 .6$

\begin{abstract}
The efficacy of adjuvant chemotherapy with S-1 in patients with completely resected non-small cell lung cancer (NSCLC) has yet to be clarified, and the appropriate schedule for the adjuvant chemotherapy with $\mathrm{S}-1$ remains unknown. A phase II study was conducted to evaluate the feasibility and efficacy of adjuvant chemotherapy with S-1. Patients enrolled in this study were 20-75 years old, had pathological stage IB-IIIA NSCLC, and had received complete resection of NSCLC. S-1 $\left(80 \mathrm{mg} / \mathrm{m}^{2}\right)$ was administered orally to the patients for four weeks followed by a two-week rest period (conventional schedule), for a maximum of eight cycles. The primary endpoint was relative dose intensity (RDI), while the secondary endpoints were safety and 1 year of disease-free survival (1y-DFS). Between May 2007 and October 2009, 28 patients were enrolled. The RDI was $63.1 \%$ (95\% CI, 48.6-77.7). No grade 3 or worse hematological toxicity was observed. Grade 3 non-hematological toxicities were observed in four patients. No grade 4 or worse hematological toxicity was detected. The probability of $1 \mathrm{y}-\mathrm{DFS}$ was $85.7 \%$ (95\% CI, 72.8-98.6). In the subgroup analysis, the median RDI of patients over 65 years old was lower compared to the other patients (44.8 vs. $100 \%$; $\mathrm{P}=0.013$; Mann-Whitney U test). Creatinine clearance $(\mathrm{CCr})$ was lower in the older group, with more grade 2 or 3 non-hematological toxicities in the elderly patients. These results suggest that the conventional schedule of adjuvant chemotherapy with S-1 is not likely to be feasible in older patients with completely resected NSCLC.
\end{abstract}

Correspondence to: Dr Shunsuke Okumura, Respiratory Center, Asahikawa Medical University, 2-1-1-1 Midorigaoka-higashi, Asahikawa, Hokkaido 0788510, Japan

E-mail: okumura-@asahikawa-med.ac.jp

Key words: non-small cell lung cancer, adjuvant chemotherapy, S-1, feasibility study

\section{Introduction}

Patients with completely resected non-small cell lung cancer (NSCLC) are at risk of relapse. Distant recurrence is a leading form of relapse in $15 \%$ of stage IA and in $60 \%$ of stage IIIA NSCLC patients (1). Previous meta-analyses of adjuvant chemotherapies have shown that the chemotherapies have survival benefit in patients with completely resected NSCLC $(2,3)$. Cisplatin (CDDP)-based chemotherapies are recommended as the adjuvant chemotherapy in surgically resected stage II and IIIA NSCLC (4,5). Among the CDDP-based combination chemotherapies, chemotherapy with CDDP and vinorelbine haveproven to be beneficial for survival in NSCLC (6). Kato et al (7) and Hamada et al (8) demonstrated that tegafur-uracil (UFT) adjuvant therapy improved the overall survival period in stage I lung adenocarcinoma, however, this result has not yet been proven worldwide.

Adjuvant chemotherapies have increased survival rates by approximately $5 \%$ in 5 years in NSCLC, which does not appear to be satisfactory $(3,9)$. Severe toxicities are occasionally observed in patients treated with CDDP-based chemotherapies, and chemotherapy-related death has been one of the major problems with adjuvant chemotherapies. Accordingly, development of a novel therapeutic strategy that is both safe and efficient is essential for patients with completely resected NSCLC.

$\mathrm{S}-1$ is an oral 5-fluorouracil-(5-FU)-based agent containing tegafur, a 5-FU pro-drug, 5-chloro-2,4-dihydroxypyridine (CDHP), and potassium oxonate (Oxo) (10). CDHP is an inhibitor of dihydropyrimidine dehydrogenase, which degrades 5-FU. Oxo is distributed in the gastrointestinal tract and inhibits orotate phosphoribosyltransferase, a phosphoenzyme for 5-FU. Adjuvant chemotherapy with the single-agent S-1 has been proven to have a survival benefit in gastric cancer patients with a gastrectomy (11). S-1 is administered to patients for four weeks followed by a two-week rest period (conventional schedule). A modified schedule, in which S-1 is administered for two weeks followed by a one-week rest period (modified schedule), is used if patients on the conventional regimen experience severe toxicities (12). 
Kim et al (13) reported that gastrectomy affected the pharmacokinetics of S-1 in patients with gastric cancer. By contrast, Kochi et al (14) demonstrated that gastrectomy had no effect on the plasma concentrations of 5-FU in patients treated with S-1. The effect of gastrectomy on the pharmacokinetics of S-1 remains controversial, while the pharmacokinetics of S-1 in patients with resected NSCLC is likely to be different compared to patients with resected gastric cancer.

In patients with advanced NSCLC, single-agent S-1 (conventional schedule) has been demonstrated to have a $22 \%$ response rate and to add 10.5 months to the median survival period (15). The feasibility of S-1 therapy was favorable to patients. Yano et al (16) reported that adjuvant chemotherapy with S-1 was feasible in patients with completely resected NSCLC. In that study, however, the conventional schedule of S-1 administration was not employed, and the schedule was modified. The feasibility of the conventional schedule of S-1 has, therefore, not previously evaluated in patients with completely resected NSCLC.

Considering that, as described above, the conventional administration schedule of S-1 has demonstrated efficacy in the treatment of advanced NSCLC (15), we hypothesized that the conventional schedule is likely to be efficient and feasible in patients with completely resected NSCLC. Consequently, the present study was conducted to evaluate the safety and efficacy of the conventional schedule of S-1 adjuvant chemotherapy in patients with completely resected NSCLC.

\section{Materials and methods}

Study design. The present study was designed as a multi-center, single-arm, clinical phase II study to evaluate the feasibility of S-1 adjuvant chemotherapy in patients with completely resected NSCLC, and was undertaken between May 2007 and October 2010 in Japan. The primary endpoint was relative dose intensity (RDI), representing the actual dose-planned dose ratio of S-1. The secondary endpoints were the safety and probability of 1-year disease-free survival (1y-DFS). The present study was designed to have $90 \%$ power for testing that RDI was not $<50 \%$ at a one-side $5 \%$ significance level, if the actual RDI was $>80 \%$. Based on that design, the minimum sample size was calculated to be 21 patients, using the mathematical formula of one-stage phase II design of the South West Oncology Group (http://www.swogstat.org/statoolsout.html). Thirty patients were scheduled to enroll in the present study, while the study population was selected from three hospitals in Hokkaido, Japan. The present study was conducted in accordance with the Declaration of Helsinki. The protocol was approved by the local Institutional Review Board.

Patients. The present study comprised patients, who underwent curative resection with ND2a lymphadenectomy for NSCLC between 2007 and 2009, with diagnoses of pathological stage IB-IIIA NSCLC. The patients provided their signed written informed consent.

Eligible patients i) were 20-75 years old, ii) had an Eastern Cooperative Oncology Group (ECOG) performance status of 0 or 1 , iii) had adequate organ function [leukocyte count $>3.5 \times 10^{9}$ cells $/ 1$, platelet count $>100 \times 10^{9}$ cells $/ 1$, hemoglobin $>9.0 \mathrm{~g} / \mathrm{dl}$, aspartate and alanine aminotransferases
(ASTs and ALTs) $<2.0 \times$ upper limit of normal (ULN), serum bilirubin $<2.0 \times \mathrm{ULN}$, serum creatinine $<1.0 \times \mathrm{ULN}$, BUN $<25 \mathrm{mg} / \mathrm{dl}$, creatinine clearance $(\mathrm{CCr}$, which was calculated by the Cockcroft-Gault equation) $>60 \mathrm{ml} / \mathrm{min}$, $\mathrm{PaO}_{2}>70 \mathrm{mmHg}$ ], iv) had undergone no prior chemotherapy for NSCLC, v), were within 8 weeks of surgery and vi ) had a life expectancy of $>3$ months.

Treatment. S-1 was administered to patients within 8 weeks of surgery. Patients received $40 \mathrm{mg} / \mathrm{m}^{2} \mathrm{~S}-1$ orally twice daily; the dose was $80 \mathrm{mg} /$ body/day when the body surface area was $<1.25 \mathrm{~m}^{2}, 100 \mathrm{mg} /$ body/day for $1.25-1.50 \mathrm{~m}^{2}$ and $120 \mathrm{mg} / \mathrm{body} /$ day for $>1.50 \mathrm{~m}^{2}$. S-1 was administered for 4 weeks followed by a two-week rest period. These cycles were repeated every 6 weeks, while the maximum treatment was 8 cycles.

Administration of S-1 was delayed if a patient had any of the following toxicities on Day 1: leukocyte count $<3.0 \times 10^{9}$ cells $/ 1$, neutrophil count $<1.5 \times 10^{9}$ cells $/ 1$, platelet count $<100 \times 10^{9}$ cells $/ 1$, hemoglobin $<8.0 \mathrm{~g} / \mathrm{dl}$, ASTs and ALTs $>2.0 \times$ ULN, serum bilirubin $>2.0 \times$ ULN, serum creatinine $>1.0 \times$ ULN or other non-hematological toxicities $>$ grade 2 .

The conventional schedule of S-1 was replaced by the modified schedule, consisting of a two-week administration of S-1 and a one-week rest period, when a patient had one of the following toxicities: leukocyte count $<2.0 \times 10^{9}$ cells/1, neutrophil count $<1.0 \times 10^{9}$ cells $/ 1$, platelet count $<75 \times 10^{9}$ cells $/ 1$, hemoglobin $<8.0 \mathrm{~g} / \mathrm{dl}$, ASTs and ALTs $>2.0 \times$ ULN, serum bilirubin $>2.0 \times$ ULN, serum creatinine $>1.0 \times$ ULN or other non-hematological toxicities $>$ grade 3 . In the modified schedule, the cycles were repeated every three weeks, while 2 cycles of the modified schedule were considered to be equivalent to 1 cycle of the conventional regimen.

When patients had suffered from the above-mentioned toxicities twice, the $\mathrm{S}-1$ dose was reduced to $50 \mathrm{mg} / \mathrm{body} /$ day for $<1.25 \mathrm{~m}^{2}, 80 \mathrm{mg} /$ body/day for $1.25-1.5 \mathrm{~m}^{2}$ and $100 \mathrm{mg} /$ body/day for $>1.5 \mathrm{~m}^{2}$. Patients received $\mathrm{S}-1$ chemotherapy unless experiencing a relapse or was experienced the following discontinuation criteria presented: i) suffered from a third round of the indicated toxicities, ii) grade 4 hematological toxicities, iii) the creatinine increased to grade 2 or worse, iv) ASTs and ALTs increased to grade 3 or worse, v) other non-hematological toxicities at grade 4 or vi) the next chemotherapy cycle was delayed for $>28$ days.

Assessment of patients. Pathological stages of NSCLC were determined using the 6 th edition of the Union for International Cancer Control Manual of Clinical Oncology (17). Toxicities were assessed according to the National Cancer Institute Common Terminology Criteria for Adverse Events version 3.0 (http://ctep.cancer.gov/reporting/ctc.html) (18). Adverse events were evaluated and blood tests were carried out at each cycle. Screening tests included hematology, blood chemistry and urinalysis. Disease-free survival (DFS) was determined by the time period between enrollment and recurrence. Computed tomography and tumor marker tests were performed at least every 6 months subsequent to enrollment. DFS in each patient was analyzed 1 year after surgery.

Statistical analysis. In the present study, P-values and confidence intervals (CI) were two-sided, and $\mathrm{P}<0.05$ was 


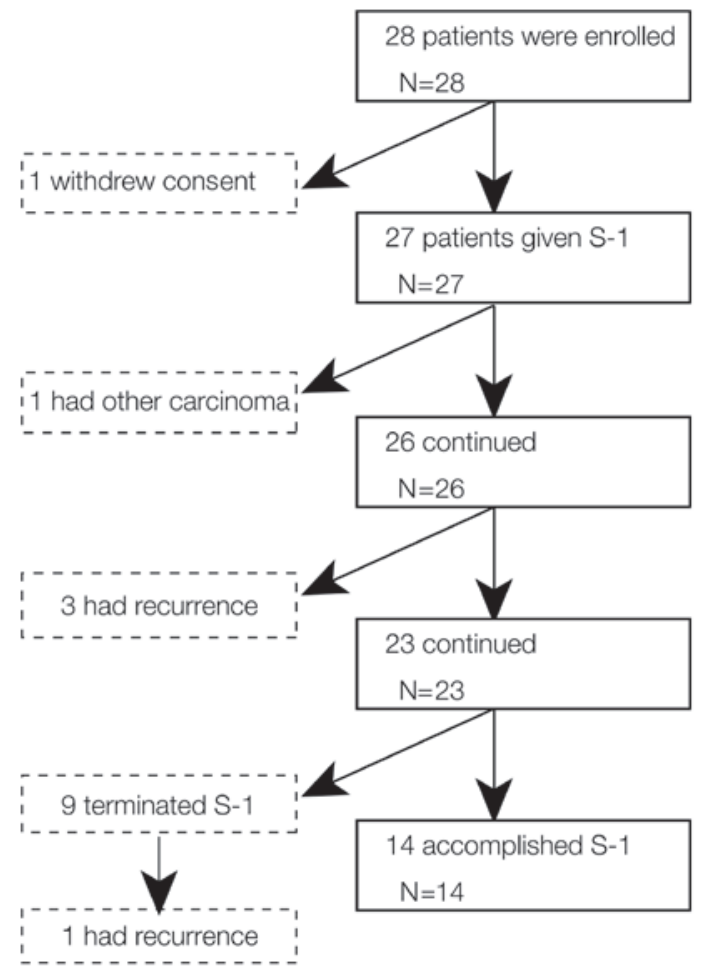

Figure 1. CONSORT diagram is shown. Of the 28 patients enrolled, 14 completed the planned 8 courses of adjuvant chemotherapy with S-1.

considered to indicate a statistically significant difference. In the subgroup analysis, the median values of RDI were compared using the Mann-Whitney U test. The probability of 1y-DFS was estimated using the Kaplan-Meier method. The correlation between the two variables (RDI and age, RDI and $\mathrm{CCr}$ ) was analyzed using the Spearman's rank correlation test. The difference between $\mathrm{CCr}$ in older ( $>65$ years) and younger $(<65$ years) groups was analyzed using the Student's t-test. Statistical analysis was carried out using SPSS Statistics version 19.0.0 (IBM, Tokyo, Japan).

\section{Results}

Patient characteristics. Between May 2007 and October 2009, 28 patients (22 male and 6 female) were enrolled in the present study (Fig. 1 and Table I). The enrollment was terminated after interim analysis was carried out and RDI was confirmed not to meet the expectations.

In the present study, one of the 28 patients refused to be treated with S-1 after enrollment, thus never receiving S-1. This patient was included in the analysis of RDI and 1-y DFS, but excluded from the subgroup analysis. The median age of patients was 67.0 years, while $78.6 \%$ of the enrolled patients were males (Table I). In this study, 15 patients had a diagnosis of NSCLC at the pathological stage IB and 18 had adenocarcinoma.

Feasibility of $S-1$ chemotherapy. The relative dose intensity for 28 patients was $63.1 \%$ (95\% CI, 48.6-77.7). Fourteen patients $(50.0 \%)$ completed all 8 planned courses of S-1, whereas the remaining patients received an incomplete adjuvant chemotherapy. The average number of completed
Table I. Patient characteristics.

\begin{tabular}{lr}
\hline Characteristics & Value \\
\hline Age (years) & \\
Median & 67.0 \\
Range & $51-75$ \\
Gender (n=28) & \\
Male & 22 \\
Female & 6 \\
Pathological stage & \\
IB & 15 \\
IIA & 3 \\
IIB & 5 \\
IIIA & 5 \\
ECOG-PS & \\
0 & 6 \\
1 & 22 \\
Histological type & \\
Adenocarcinoma & 18 \\
Squamous cell carcinoma & 7 \\
Others & 3
\end{tabular}

aEastern Cooperative Oncology Group Performance Status.

Table II. Toxicities.

Event Grade 1 Grade 2 Grade 3 Grade 4

\section{Hematological \\ Leukopenia \\ Neutropenia \\ Anemia \\ Platelet}

Non-hematological

$\begin{array}{lrrrr}\text { Skin hyperpigmentation } & 15 & 0 & 0 & 0 \\ \text { Rash } & 3 & 2 & 0 & 0 \\ \text { Diarrhea } & 10 & 1 & 0 & 0 \\ \text { Nausea } & 3 & 4 & 0 & 0 \\ \text { Anorexia } & 13 & 4 & 1 & 0 \\ \text { AST or ALT } & 7 & 2 & 0 & 0 \\ \text { Creatinine } & 1 & 1 & 0 & 0 \\ \text { Fatigue } & 13 & 3 & 0 & 0 \\ \text { Dysgeusia } & 2 & 1 & 0 & 0 \\ \text { Oral mucositis } & 1 & 0 & 0 & 0 \\ \text { Peripheral neuropathy } & 1 & 1 & 0 & 0 \\ \text { Pyramidal syndrome } & 0 & 0 & 1 & 0 \\ \text { Pneumonitis } & 0 & 0 & 1 & 0 \\ \text { Serum K increased } & 1 & 1 & 0 & 0 \\ \text { Serum AMY increased } & 0 & 0 & 1 & 0 \\ \text { Dizziness } & 1 & 0 & 0 & 0\end{array}$

Table shows no. of patients. AST, aspartate aminotransferase; ALT, alanine aminotransferase; AMY, amylase. 


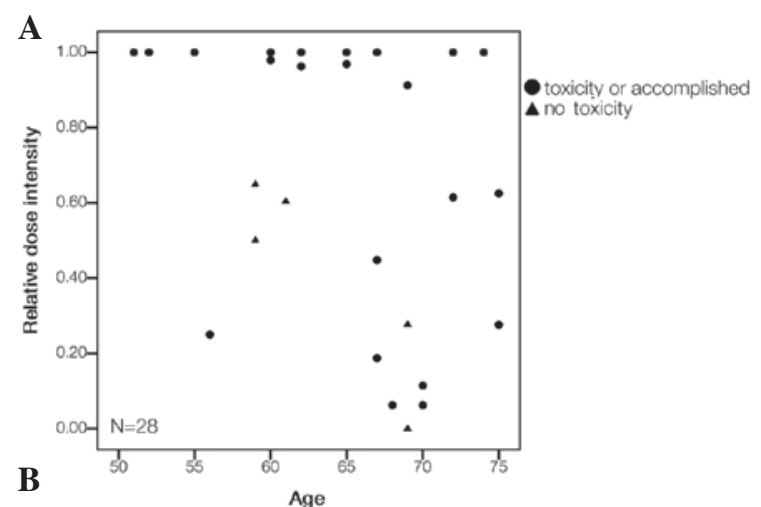

A

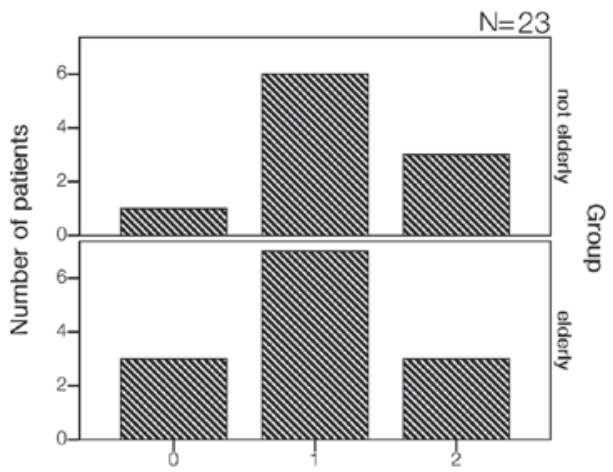

The worst grade of hematological toxicity
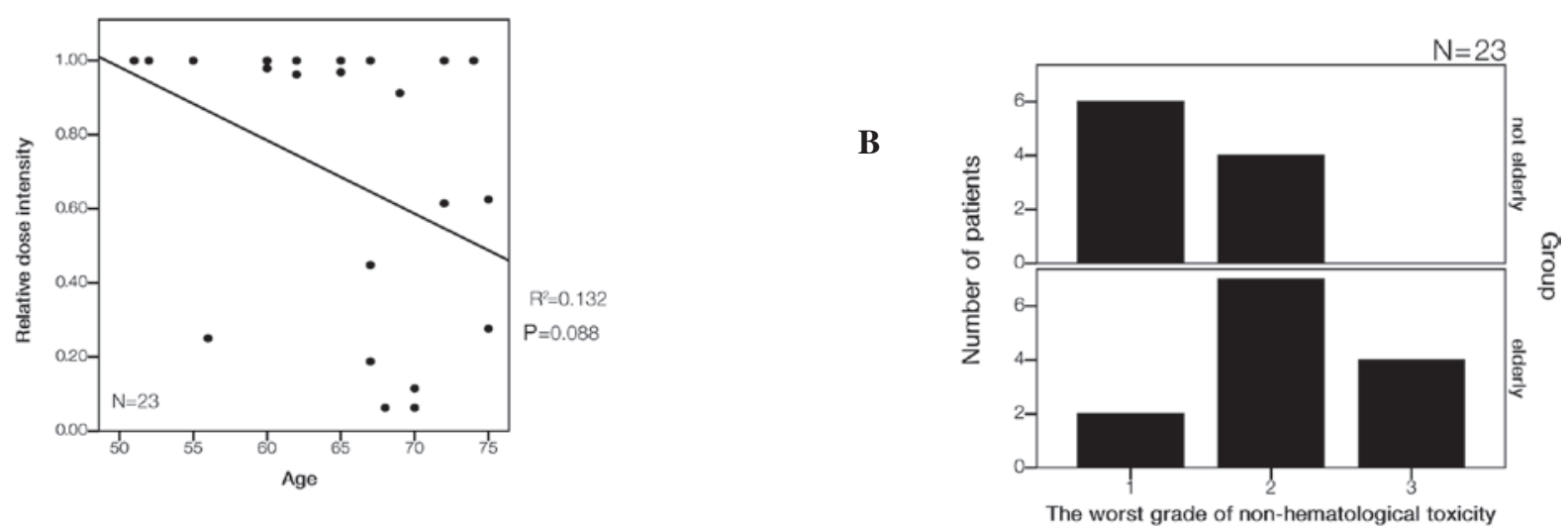

Figure 2. Scatter diagram of relative dose intensity (RDI) and age is shown. (A) No correlation between RDI and age in 28 patients was detected. (B) RDI seemed to differ in 23 patients, according to age $\left(\mathrm{R}^{2}=0.132 ; \mathrm{P}=0.088\right.$; Spearman's rank correlation test). In the analysis, 5 patients without toxicityrelated discontinuation (represented as triangle markers) were excluded (A).

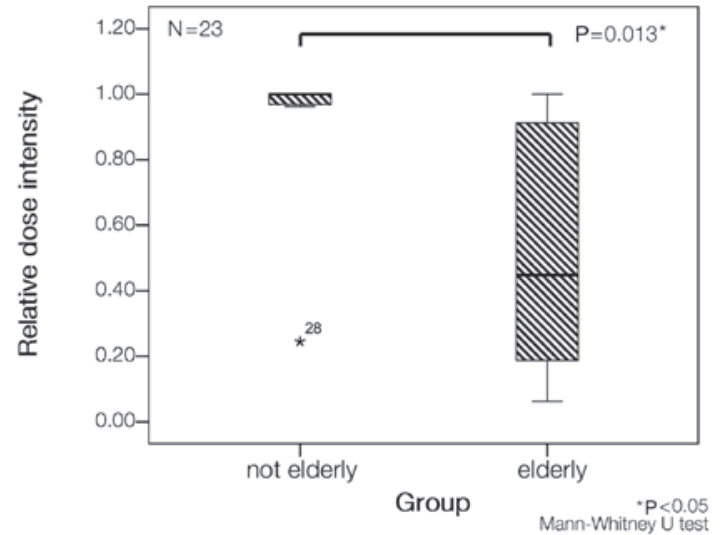

Figure 3. Relative dose intensity (RDI) in the older ( $>65$ years) and younger groups ( $\leq 65$ years) is shown. The median RDI in older patients was significantly lower than that in younger patients ( 44.8 vs. $100 \%$; $\mathrm{P}=0.013$; Mann-Whitney U test).

courses was 5.3 (95\% CI, 4.1-6.5). A schedule modification was required for 10 patients (35.7\%). Additionally, the dose was reduced for $7(70.0 \%)$ of the 10 patients, requiring a schedule modification.

Fig. 2A shows a scatter diagram of the correlation between age and RDI in the 28 patients. Fig. 2B shows a scatter diagram of 23 patients, while 5 patients were excluded from the analysis for reasons other than toxicities: 4 patients terminated chemotherapy due to a recurrence or a simultaneous carcinoma; 1 patient refused to receive S-1 chemotherapy (excluded patients are represented as triangles in Fig. 2A). Considering the distribution in the scatter diagram, the RDI seemed to be lower in patients $>65$ years $\left(\mathrm{R}^{2}=0.132 ; \mathrm{P}=0.088\right.$; Spearman's rank correlation test) (Fig. 2B). The patients were divided into groups of older (age $>65$ years) and younger patients (age $\leq 65$ years), showing that the median RDI in the older 


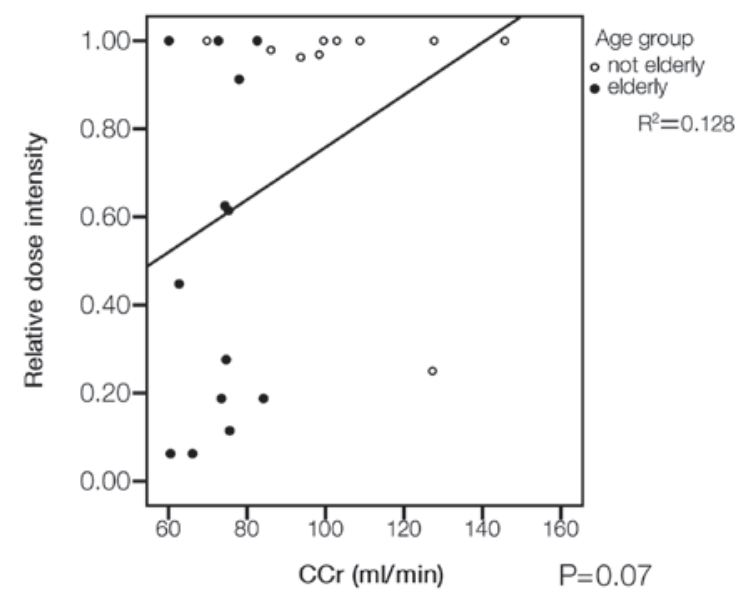

Figure 6. Relative dose intensity (RDI) and creatinine clearance $(\mathrm{CCr})$ is shown. CCr seemed to be correlated with RDI.

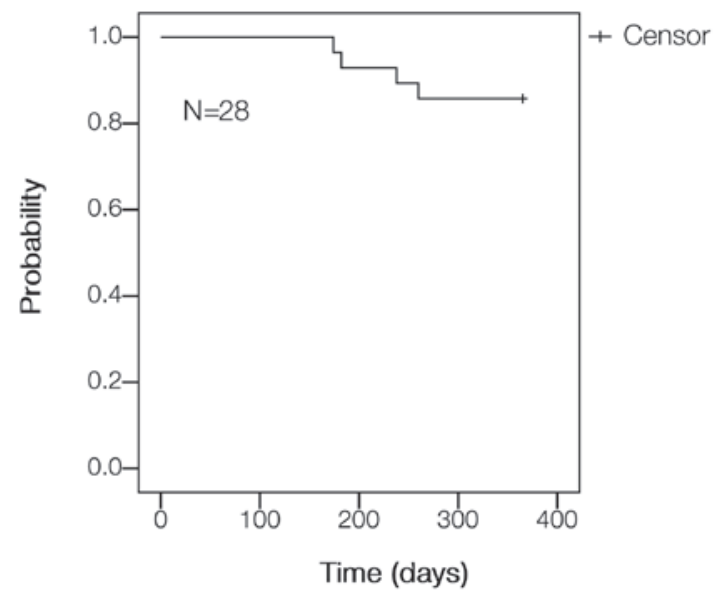

Figure 7. The probability of 1-year disease-free survival was $85.7 \%$ (95\% CI, 72.8-98.6).

patients was markedly lower compared to the younger patients (44.8 vs. 100\%; P=0.013; Mann-Whitney U test) (Fig. 3).

Toxicity profiles. Adverse events in 27 eligible patients were analyzed. No grade 4 or 5 toxicity was observed (Table II), while 4 patients had grade 3 adverse events including anorexia, extrapyramidal disorder, pneumonitis and elevated serum amylase. In particular, $3 / 4$ of patients had grade 3 non-hematological toxicities on Days 15-29. No grade 3 or worse hematological toxicity was observed in the present study. The most common adverse events were anorexia, fatigue, diarrhea and skin hyperpigmentation.

When the patients were divided by age as described above, grade 2 and 3 non-hematological events were more likely to be observed in the older compared to the younger group (Fig. 4). By contrast, the frequencies of hematological toxicities in the older and younger groups were similar. To determine the factor responsible for those differences, $\mathrm{CCr}$ was analyzed in each group. CCr values in older patients were lower compared to younger patients (Fig. 5), while $\mathrm{CCr}$ appeared to be correlated with RDI ( $\mathrm{P}=0.07 ; \mathrm{R}^{2}=0.128$; Spearman's rank correlation test) (Fig. 6).

One-year DFS. The probability of DFS at 1 year was $85.7 \%$ (95\% CI, 72.8-98.6) (Fig. 7). The average of 1y-DFS period was 343.4 days (95\% CI, 323.1-363.6). Relapse was observed in 4 patients $(14.3 \%)$. Of the 4 patients, 2 had stage IB NSCLC and 2 had stage IIB and IIIA.

\section{Discussion}

In the present study, the feasibility of chemotherapy with S-1 in patients with completely resected NSCLC was evaluated. When a novel agent is employed in an adjuvant chemotherapy, both efficacy and safety are of great interest. S-1 is an oral agent that was administered on an outpatient basis. It is reasonable to use S-1 in adjuvant chemotherapy of NSCLC, if S-1 has a favorable feasibility and efficacy equivalent to that of platinum-based chemotherapy. In the present study, four major findings were reported: i) the average of RDI was $63.1 \%$, while a low S-1 RDI was observed in patients $>65$ years, ii) no grade 4 or worse toxicity was observed, although the older subgroup was more likely to have non-hematological grade 2 and 3 toxicities, iii) low $\mathrm{CCr}$ was observed in the older patients, possibly accounting for both the increase in non-hematological toxicities and the low RDI in the older patients and v) the $86 \%$ probability of $1 \mathrm{y}$-DFS in the present study was acceptable.

In the present study, the RDI of S-1 was $63.1 \%$, although only $50 \%$ of the patients completed chemotherapy with $\mathrm{S}-1$, which was not satisfactory. In gastric cancer, Sakuramoto et al (11) demonstrated the survival benefit of adjuvant chemotherapy with S-1, as delineated above. In their large-scale study, 66\% of patients completed chemotherapy with S-1, while $40 \%$ of patients required modification of the treatment schedule $(11,12)$. The RDI in the present study seemed to be inferior to that in the large-scale study.

The subgroup analysis of our results revealed that patients $>65$ years had a markedly lower RDI compared to the remaining patients (Figs. 2 and 3). Aoyama et al (19) reported that in gastric cancer no difference was observed in the RDI of the adjuvant chemotherapy with $S-1$ in older ( $\geq 70$ years old) and younger ( $<70$ years old) patients with gastrectomy. However, Tsushima et al (20) demonstrated that median RDI in younger ( $\leq 65$ years old) patients was likely to be lower compared to older ( $>65$ years old) patients, including patients with unresectable gastric cancer. The conventional schedule of S-1 administration was employed in the two studies. Since the present study comprised no patients with gastrectomy, we referred to the results reported by Tsushima et al (20). These results suggest that age is likely to affect RDI in the conventional S-1 schedule in NSCLC.

Moreover, no grade 4 or worse toxicity was observed in the present study (Table II). The present toxicity profile appeared to be comparable to other studies, suggesting that S-1 may be used in outpatients with completely resected NSCLC. However, results of the present study suggest that the older patients were more likely to have grade 3 non-hematological toxicities, while the incidence of hematological toxicities was similar (Fig. 4). 
In addition, lower $\mathrm{CCr}$ in elderly patients was likely to be associated with lower RDI (Figs. 5 and 6). Tsushima et al (20) reported that $\mathrm{CCr}$ affected the dose intensity of $\mathrm{S}-1$ in advanced gastric cancer (12). Several studies have demonstrated that approximately $50 \%$ of CDHP, a component of S-1 preventing degradation of 5-FU, was excreted in urine, and that renal function affects 5-FU concentrations in patients with solid tumors (21-23). Moreover, Fujita et al (24) reported that the area under the curve for CDHP was significantly higher in older ( $\geq 75$ years) compared to younger patients, due to reduced renal function. Accordingly, we hypothecized that lower $\mathrm{CCr}$ in older patients is likely to have affected the toxicity profile and RDI in the present study.

In order to increase RDI in elderly patients, we referred to studies in which S-1 schedule or dose modification was employed. Several studies demonstrated that schedule- or dosemodified S-1 therapy increased RDI in adjuvant chemotherapy with S-1 (25-28). Shin et al (29) reported a modified schedule in which S-1 was administered for two weeks followed by a one-week rest period to be preferable for older patients with advanced colorectal cancer. However, a phase I study of S-1 demonstrated that the recommended dose of S-1 was $30 \mathrm{mg} / \mathrm{m}^{2}$ for North American patients with advanced upper gastrointestinal cancer (30). Yano et al (16) demonstrated that in patients with resected NSCLC, the modified schedule of S-1 generated a preferable RDI (73.9\%), as delineated above. Currently, most patients with NSCLC are $>65$ years, and a schedule or dose modification may be preferable for the adjuvant chemotherapy with S-1.

In the present study, the probability of 1y-DFS was $85.7 \%$ (Fig. 7), thus not lower than the outcome reported in the post-operative course (31). However, this study included patients with stage IB-IIIA NSCLC. We hypothesized that eligibility might slightly affect the probability of 1y-DFS, although the sample size was too small to estimate the survival benefit of chemotherapy with S-1. As described above, single-agent $\mathrm{S}-1$ has been shown to provide a preferable response rate in advanced NSCLC, although the adverse effects appear to be slightly less severe compared to those of platinum-based chemotherapy, including combination chemotherapy with a platinum agent and S-1 (15,32-35). Thus, single-agent S-1 may be appropriate for patients with stage I NSCLC.

In conclusion, the present data suggest that single-agent S-1 in adjuvant chemotherapy often requires schedule or dose modification. The conventional S-1 schedule is not likely to be feasible in patients with completely resected NSCLC.

\section{Acknowledgements}

The authors thank Drs Yoshinori Minami, Satoshi Endoh, Satoshi Hayashi, Kiyoko Shibukawa, Kaori Ohnishi (Respiratory Center, Asahikawa Medical University, Asahikawa, Japan), Drs Kensuke Ishida, Shoko Takeuchi, Eri Toyoshima, Yasushi Yamamoto, Toshiyuki Ogasa, Shinobu Osanai (First Department of Internal Medicine, Asahikawa Medical University, Asahikawa, Japan), Drs Satoshi Hirata (Department of Operation, Asahikawa Medical University), and Dr Hiroyuki Aoki (Department of Respiratory Surgery, National Hospital Organization,
Asahikawa Medical Center, Asahikawa, Japan) for their support and for the enrollment of patients in the present study.

\section{References}

1. Pisters KM and Le Chevalier T: Adjuvant chemotherapy in completely resected non-small-cell lung cancer. J Clin Oncol 23: 3270-3278, 2005

2. Pignon JP, Tribodet H, Scagliotti GV, et al: Lung adjuvant cisplatin evaluation: a pooled analysis by the LACE Collaborative Group. J Clin Oncol 26: 3552-3559, 2008.

3. Arriagada R, Auperin A, Burdett S, et al: Adjuvant chemotherapy, with or without postoperative radiotherapy, in operable non-small-cell lung cancer: two meta-analyses of individual patient data. Lancet 375: 1267-1277, 2010.

4. Pisters KM, Evans WK, Azzoli CG, et al: Cancer Care Ontario and American Society of Clinical Oncology adjuvant chemotherapy and adjuvant radiation therapy for stages I-IIIA resectable non small-cell lung cancer guideline. J Clin Oncol 25: 5506-5518, 2007.

5. Crino L, Weder W, van Meerbeeck J and Felip E: Early stage and locally advanced (non-metastatic) non-small-cell lung cancer: ESMO Clinical Practice Guidelines for diagnosis, treatment and follow-up. Ann Oncol 21: v103-v115, 2010.

6. Douillard JY, Tribodet H, Aubert D, et al: Adjuvant cisplatin and vinorelbine for completely resected non-small cell lung cancer: subgroup analysis of the Lung Adjuvant Cisplatin Evaluation. J Thorac Oncol 5: 220-228, 2010.

7. Kato H, Ichinose $\mathrm{Y}$, Ohta M, et al: A randomized trial of adjuvant chemotherapy with uracil-tegafur for adenocarcinoma of the lung. N Engl J Med 350: 1713-1721, 2004.

8. Hamada C, Tsuboi M, Ohta M, et al: Effect of postoperative adjuvant chemotherapy with tegafur-uracil on survival in patients with stage IA non-small cell lung cancer: an exploratory analysis from a meta-analysis of six randomized controlled trials. J Thorac Oncol 4: 1511-1516, 2009.

9. Fruh M, Rolland E, Pignon JP, et al: Pooled analysis of the effect of age on adjuvant cisplatin-based chemotherapy for completely resected non-small-cell lung cancer. J Clin Oncol 26: 3573-3581, 2008.

10. Shirasaka T, Nakano K, Takechi T, et al: Antitumor activity of $1 \mathrm{M}$ tegafur-0.4 M 5-chloro-2,4-dihydroxypyridine-1 M potassium oxonate (S-1) against human colon carcinoma orthotopically implanted into nude rats. Cancer Res 56: 2602-2606, 1996.

11. Sakuramoto S, Sasako M, Yamaguchi T, et al: Adjuvant chemotherapy for gastric cancer with S-1, an oral fluoropyrimidine. N Engl J Med 357: 1810-1820, 2007.

12. Iwasa S, Yamada Y, Fukagawa T, et al: Management of adjuvant S-1 therapy after curative resection of gastric cancer: dose reduction and treatment schedule modification. Gastric Cancer 14: 28-34, 2011.

13. Kim WY, Nakata B and Hirakawa K: Alternative pharmacokinetics of S-1 components, 5-fluorouracil, dihydrofluorouracil and alpha-fluoro-beta-alanine after oral administration of S-1 following total gastrectomy. Cancer Sci 98: 1604-1608, 2007.

14. Kochi M, Fujii M, Kanamori N, et al: Effect of gastrectomy on the pharmacokinetics of S-1, an oral fluoropyrimidine, in resectable gastric cancer patients. Cancer Chemother Pharmacol 60: 693-701, 2007.

15. Kawahara M, Furuse K, Segawa Y, et al: Phase II study of S-1, a novel oral fluorouracil, in advanced non-small-cell lung cancer. Br J Cancer 85: 939-943, 2001.

16. Yano T, Yamazaki K, Maruyama R, et al: Feasibility study of postoperative adjuvant chemotherapy with S-1 (tegaful, gimeracil, oteracil potassium) for non-small cell lung cancerLOGIK 0601 study. Lung Cancer 67: 184-187, 2010.

17. Sobin L and Witthekind C: TNM Classification of Malignant Tumours, 6th edition. Wiley-Liss, New York, pp99-103, 2002.

18. Trotti A, Colevas AD, Setser A, et al: CTCAE v3.0: development of a comprehensive grading system for the adverse effects of cancer treatment. Semin Radiat Oncol 13: 176-181, 2003.

19. Aoyama T, Yoshikawa T, Watanabe T, et al: Safety and feasibility of S-1 adjuvant chemotherapy for gastric cancer in elderly patients. Gastric Cancer 15: 76-82, 2011.

20. Tsushima T, Hironaka S, Boku N, et al: Safety and efficacy of S-1 monotherapy in elderly patients with advanced gastric cancer. Gastric Cancer 13: 245-250, 2010. 
21. Hirata K, Horikoshi N, Aiba K, et al: Pharmacokinetic study of S-1, a novel oral fluorouracil antitumor drug. Clin Cancer Res 5: 2000-2005, 1999.

22. Fujita K, Yamamoto W, Endo S, et al: CYP2A6 and the plasma level of 5-chloro-2, 4-dihydroxypyridine are determinants of the pharmacokinetic variability of tegafur and 5-fluorouracil, respectively, in Japanese patients with cancer given S-1. Cancer Sci 99: 1049-1054, 2008.

23. Ikeda M, Furukawa H, Imamura H, et al: Pharmacokinetic study of S-1, a novel oral fluorouracil antitumor agent in animal model and in patients with impaired renal function. Cancer Chemother Pharmacol 50: 25-32, 2002.

24. Fujita K, Nakayama H, Ichikawa W, et al: Pharmacokinetics of 5 -fluorouracil in elderly Japanese patients with cancer treated with S-1 (a combination of tegafur and dihydropyrimidine dehydrogenase inhibitor 5-chloro-2,4-dihydroxypyridine). Drug Metab Dispos 37: 1375-1377, 2009.

25. Kimura Y, Kikkawa N, Iijima S, et al: A new regimen for S-1 therapy aiming at adverse reaction mitigation and prolonged medication by introducing a 1 -week drug-free interval after each 2-week dosing session: efficacy and feasibility in clinical practice. Gastric Cancer 6: 34-39, 2003.

26. Tsukuda M, Kida A, Fujii M, et al: Randomized scheduling feasibility study of S-1 for adjuvant chemotherapy in advanced head and neck cancer. Br J Cancer 93: 884-889, 2005.

27. Sakuma K, Hosoya Y, Arai W, et al: Alternate-day treatment with S-1 in patients with gastric cancer: a retrospective study of strategies for reducing toxicity. Int J Clin Oncol 15: 166-171, 2010.

28. Arai W, Hosoya Y, Hyodo M, et al: Alternate-day oral therapy with TS-1 for advanced gastric cancer. Int J Clin Oncol 9 : 143-148, 2004
29. Shin SJ, Jeong JH, Park YS, et al: Phase II trial of S-1 monotherapy in elderly or frail patients with metastatic colorectal cancer. Invest New Drugs 29: 1073-1080, 2010.

30. Zhu AX, Clark JW, Ryan DP, et al: Phase I and pharmacokinetic study of S-1 administered for 14 days in a 21-day cycle in patients with advanced upper gastrointestinal cancer. Cancer Chemother Pharmacol 59: 285-293, 2007.

31. Hanagiri T, Baba T, So T, et al: Time trends of surgical outcome in patients with non-small cell lung cancer. J Thorac Oncol 5: 825-829, 2010.

32. Schiller JH, Harrington D, Belani CP, et al: Comparison of four chemotherapy regimens for advanced non-small-cell lung cancer. N Engl J Med 346: 92-98, 2002.

33. Ohe Y, Ohashi Y, Kubota K, et al: Randomized phase III study of cisplatin plus irinotecan versus carboplatin plus paclitaxel, cisplatin plus gemcitabine, and cisplatin plus vinorelbine for advanced non-small-cell lung cancer: Four-Arm Cooperative Study in Japan. Ann Oncol 18: 317-323, 2007.

34. Okamoto I, Yoshioka H, Morita S, et al: Phase III trial comparing oral S-1 plus carboplatin with paclitaxel plus carboplatin in chemotherapy-naive patients with advanced non-small-cell lung cancer: results of a west Japan oncology group study. J Clin Oncol 28: 5240-5246, 2010.

35. Kubota K, Sakai H, Yamamoto N, et al: A multi-institution phase I/II trial of triweekly regimen with $\mathrm{S}-1$ plus cisplatin in patients with advanced non-small cell lung cancer. J Thorac Oncol 5: 702-706, 2010. 